\title{
Interpreting LHC SUSY searches in the phenomenological MSSM
}

\author{
S. Sekmen, ${ }^{a}$ S. Kraml, ${ }^{b}$ J. Lykken, ${ }^{c}$ F. Moortgat, ${ }^{d}$ S. Padhi, ${ }^{e}$ L. Pape, ${ }^{d}$ M. Pierini, ${ }^{f}$ \\ H.B. Prosper $^{a}$ and M. Spiropulu ${ }^{f, g}$ \\ ${ }^{a}$ Department of Physics, Florida State University, \\ Tallahassee, Florida 32306, U.S.A. \\ ${ }^{b}$ Laboratoire de Physique Subatomique et de Cosmologie, UJF Grenoble 1, \\ CNRS/IN2P3, INPG, 53 Avenue des Martyrs, F-38026 Grenoble, France \\ ${ }^{c}$ Fermi National Accelerator Laboratory, \\ P.O. Box 500, Batavia, IL 60510, U.S.A. \\ ${ }^{d}$ Institute for Particle Physics, \\ ETH Zurich, CH-8093 Zurich, Switzerland \\ e University of California, \\ San Diego, CA 92093, U.S.A. \\ ${ }^{f} C E R N$, \\ CH-1211 Geneva 23, Switzerland \\ ${ }^{g}$ Department of Physics, Caltech, \\ Pasadena, California 91125, U.S.A. \\ E-mail: sezen.sekmen@cern.ch, sabine.kraml@lpsc.in2p3.fr, \\ joseph.david.lykken@cern.ch, filip.moortgat@cern.ch, \\ Sanjay.Padhi@cern.ch, Luc.Pape@cern.ch, maurizio.pierini@cern.ch, \\ harry@hep.fsu.edu, Maria.Spiropulu@cern.ch
}

ABSTRACT: We interpret within the phenomenological MSSM (pMSSM) the results of SUSY searches published by the CMS collaboration based on the first $\sim 1 \mathrm{fb}^{-1}$ of data taken during the $2011 \mathrm{LHC}$ run at $7 \mathrm{TeV}$. The pMSSM is a 19-dimensional parametrization of the MSSM that captures most of its phenomenological features. It encompasses, and goes beyond, a broad range of more constrained SUSY models. Performing a global Bayesian analysis, we obtain posterior probability densities of parameters, masses and derived observables. In contrast to constraints derived for particular SUSY breaking schemes, such as the CMSSM, our results provide more generic conclusions on how the current data constrain the MSSM.

KEYWORds: Supersymmetry Phenomenology

ARXIV EPRINT: 1109.5119 


\section{Contents}

1 Introduction 1

2 Parametrization $\quad 2$

3 Analysis 3

4 Results 5

5 Conclusions $\quad 10$

\section{Introduction}

With the successful operation of the Large Hadron Collider (LHC) and its detectors in 2010-11, and with excellent prospects for the future, the LHC is ready to shed light on the most pressing open issues in particle physics: the mechanism of electroweak (EW) symmetry breaking and the nature of the new physics beyond the Standard Model that stabilizes the EW scale (see, for example, ref. [1]).

A wealth of theories that extend the Standard Model (SM) have been put forth during the past decades. Among these, supersymmetry (SUSY) is arguably the best motivated and certainly the most thoroughly studied (see, for example, refs. [2, 3] for recent reviews). Indeed, searches for SUSY rank among the primary experimental objectives of the LHC. So far, however, no signal of new physics has been observed at $\sqrt{s}=7 \mathrm{TeV}$ [4]; consequently, the SUSY mass scale has been pushed into the TeV region.

It is important to note, however, that in the interpretation of their experimental results, both the ATLAS and CMS collaborations typically use a very special theoretical model, the so-called Constrained Minimal Supersymmetric Standard Model (CMSSM), which is characterized by just four-and-a-half parameters [5-11]: a universal scalar mass $m_{0}$, gaugino mass $m_{1 / 2}$ and trilinear coupling $A_{0}$ defined at the GUT scale $M_{\mathrm{GUT}} \sim 10^{16} \mathrm{GeV}$, plus $\tan \beta$ and $\operatorname{sign}(\mu)$. The simplifying assumption of universality at the GUT scale makes the model very predictive and a convenient showcase for SUSY phenomenology. Indeed, it is interesting to present limits within the CMSSM because it provides (to some degree) an easy way to show performances, compare limits or reaches, etc... On the other hand, the interpretation of experimental results in the $\left(m_{0}, m_{1 / 2}\right)$ plane risks imposing unwarranted constraints on SUSY, as many mass patterns and signatures that are possible a priori are not covered in the CMSSM. The same of course holds true for Simplified Models [12].

In this Letter, we therefore present a more general approach, using a 19-dimensional parametrization of the MSSM called the phenomenological MSSM (pMSSM) [13]. Using results from three independent CMS analyses — the $\alpha_{T}$ hadronic [14], the same-sign 
dilepton [15] and the opposite-sign dilepton [16] analyses — we derive constraints on the SUSY particles with as few simplifying assumptions as possible.

The pMSSM parameter space has been thoroughly scanned and studied previously in refs. [17-20]. It is important to note that [17] "the pMSSM leads to a much broader set of predictions for the properties of the SUSY partners as well as for a number of experimental observables than those found in any of the conventional SUSY breaking scenarios such as mSUGRA [CMSSM]. This set of models can easily lead to atypical expectations for SUSY signals at the LHC."

The purpose of this Letter is thus to initiate a systematic study that begins with an assessment of what current LHC data tell us, and do not tell us, about the pMSSM. We choose to conduct our study using the Bayesian approach $[21,22]$ because of its conceptual coherence and the direct (intuitive) manner in which probabilistic statements are interpreted, namely, as the degree of belief, or plausibility, of a given statement. A detailed Bayesian study of the pMSSM was performed in ref. [18]; however this was before LHC data were available. ${ }^{1}$

We introduce the pMSSM and its parametrization in section 2, and outline our analysis in section 3. Our results are presented in section 4. Section 5 contains our conclusions.

\section{Parametrization}

The pMSSM, a 19-dimensional realization [13] of the R-parity conserving MSSM with parameters defined at the SUSY scale, $M_{\mathrm{SUSY}}=\sqrt{m_{\tilde{t}_{1}} m_{\tilde{t}_{2}}}$, employs only a few plausible assumptions motivated by experiment: there are no new $\mathrm{CP}$ phases, the sfermion mass matrices and trilinear couplings are flavor-diagonal, the first two generations of sfermions are degenerate and their trilinear couplings are negligible. In addition, we assume that the lightest supersymmetric particle (LSP) is the lightest neutralino, $\tilde{\chi}_{1}^{0}$. We thus arrive at a proxy for the MSSM characterized by 19 real, weak-scale, SUSY Lagrangian parameters:

- the gaugino mass parameters $M_{1}, M_{2}$, and $M_{3}$;

- the ratio of the Higgs VEVs $\tan \beta=v_{2} / v_{1}$;

- the higgsino mass parameter $\mu$ and the pseudo-scalar Higgs mass $m_{A}$;

- 10 sfermion mass parameters $m_{\tilde{F}}$, where $\tilde{F}=\tilde{Q}_{1}, \tilde{U}_{1}, \tilde{D}_{1}, \tilde{L}_{1}, \tilde{E}_{1}, \tilde{Q}_{3}, \tilde{U}_{3}, \tilde{D}_{3}, \tilde{L}_{3}, \tilde{E}_{3}$ (imposing $m_{\tilde{Q}_{1}} \equiv m_{\tilde{Q}_{2}}, m_{\tilde{L}_{1}} \equiv m_{\tilde{L}_{2}}$, etc.), and

- 3 trilinear couplings $A_{t}, A_{b}$ and $A_{\tau}$,

in addition to the SM parameters.

For each pMSSM point, we use SoftSUSY3.1.6 [24] to compute the SUSY spectrum, SuperIsov3.0 [25] to compute the low-energy constraints, micrOMEGAs2.4 [26-28] for the

\footnotetext{
${ }^{1}$ In ref. [23], the CMSSM gluino-squark mass limits based on $1 \mathrm{fb}^{-1}$ of LHC data were applied to a dark matter global fit in a 9-parameter realization of the MSSM.
} 


\begin{tabular}{|c|c|c|c|}
\hline$i$ & Observable & $\begin{array}{c}\text { Experimental result } \\
\mu_{i}\end{array}$ & $\begin{array}{c}\text { Likelihood function } \\
L\left(D_{i} \mid \mu_{i}\right)\end{array}$ \\
\hline 1 & $B R(b \rightarrow s \gamma)[33,34]$ & $(3.55 \pm 0.34) \times 10^{-4}$ & Gaussian \\
\hline 2 & $B R\left(B_{s} \rightarrow \mu \mu\right)[35]$ & $\leq 4.7 \times 10^{-8}$ & $1 /\left(1+\exp \left(\frac{\mu_{2}-D_{2}}{0.01 D_{2}}\right)\right)$ \\
\hline 3 & $R\left(B_{u} \rightarrow \tau \nu\right)[35]$ & $1.66 \pm 0.54$ & Gaussian \\
\hline 4 & $\Delta_{\mu}[36]$ & $(28.7 \pm 8.0) \times 10^{-10}\left[e^{+} e^{-}\right]$ & Weighted Gaussian average \\
\hline 5 & $m_{t}[37]$ & $173.3 \pm 1.1 \mathrm{GeV}$ & Gaussian \\
\hline 6 & $m_{b}\left(m_{b}\right)[35]$ & $4.19_{-0.06}^{+0.18} \mathrm{GeV}$ & Two-sided Gaussian \\
\hline 7 & $\alpha_{s}\left(M_{Z}\right)[38]$ & $0.1176 \pm 0.002$ & Gaussian \\
\hline 8 & $m_{h}$ & $\begin{array}{c}\text { LEP\&Tevatron } \\
(\text { HiggsBounds }[29,30])\end{array}$ & $\begin{array}{c}L_{8}^{\prime}=1 \text { if allowed. } L_{8}=10^{-9} \text { if } \\
\text { sampled from Gauss }\left(m_{h}, 1.5\right) \\
\end{array}$ \\
\hline 9 & sparticle & is excluded. \\
\hline
\end{tabular}

Table 1. The preLHC experimental results that are the basis of our pMSSM parameter scan using Markov Chain Monte Carlo (MCMC) sampling. We re-weight a posteriori with the new limit $B R\left(B_{s} \rightarrow \mu \mu\right) \leq 1.08 \times 10^{-8}$ at $95 \%$ CL [39]. However, this has hardly any effect.

SUSY mass limits, and HiggsBounds2 2.0.0 $[29,30]$ for the limit on the $h^{0}$ mass. $^{2}$ Moreover, we use SUSYHIT (SDECAY1.3b, HDECAY3.4) [31] to produce SUSY and Higgs decay tables, and micrOMEGAs2.4 [26-28] to compute the LSP relic density and direct dection cross sections. The various codes are interfaced using the SUSY Les Houches Accord [32].

\section{Analysis}

As noted in the Introduction, the purpose of this Letter is to assess what current data tell us, and do not tell us, about the pMSSM. It is convenient to partition these data into preLHC and LHC experimental results, which we list in tables 1 and 2, respectively. We use the former to construct a prior $\pi(\theta)$ on the pMSSM parameter space, which, when combined with a likelihood function, $L(\mathrm{LHC} \mid \theta)$, pertaining to the LHC results, yields the posterior density $p(\theta \mid \mathrm{LHC}) \sim L(\mathrm{LHC} \mid \theta) \pi(\theta)$ over the pMSSM parameter space. Here, $\theta$ denotes the 19 pMSSM parameters $M_{1}, \cdots, A_{\tau}$. We also consider the SM parameters $m_{t}, m_{b}\left(m_{b}\right)$ and $\alpha_{s}\left(M_{Z}\right)$, which are treated as nuisance parameters (see table 1). This

\footnotetext{
${ }^{2}$ In evaluating the Higgs mass limit, we apply a Gauss-distributed theoretical uncertainty with $\sigma=$ $1.5 \mathrm{GeV}$ to the $m_{h}$ computed by with SoftSUSY, cf. row 8 in table 1.
} 


\begin{tabular}{|c|l|c|c|}
\hline$j$ & $\begin{array}{c}\text { Analysis and search region } \\
(\text { values in } \mathrm{GeV})\end{array}$ & $\begin{array}{c}\text { Observed } \\
\text { event count } \\
\left(N_{j}\right)\end{array}$ & $\begin{array}{c}\text { Data-driven SM } \\
\text { BG estimate } \\
\left(B_{j} \pm \delta B_{j}\right)\end{array}$ \\
\hline 1 & $\alpha_{T}$ hadronic, $275 \leq H_{T}<325$ & 782 & $787.4_{-22.3}^{+31.5}$ \\
2 & $\alpha_{T}$ hadronic, $325 \leq H_{T}<375$ & 321 & $310.4_{-12.4}^{+8.4}$ \\
3 & $\alpha_{T}$ hadronic, $375 \leq H_{T}<475$ & 196 & $202.1_{-9.4}^{+8.6}$ \\
4 & $\alpha_{T}$ hadronic, $475 \leq H_{T}<575$ & 62 & $60.4_{-3.0}^{+4.2}$ \\
5 & $\alpha_{T}$ hadronic, $575 \leq H_{T}<675$ & 21 & $20.3_{-1.1}^{+1.8}$ \\
6 & $\alpha_{T}$ hadronic, $675 \leq H_{T}<775$ & 6 & $7.7_{-0.5}^{+0.8}$ \\
7 & $\alpha_{T}$ hadronic, $775 \leq H_{T}<875$ & 3 & $3.2_{-0.2}^{+0.4}$ \\
8 & $\alpha_{T}$ hadronic, $875 \leq H_{T}$ & 1 & $2.8_{-0.2}^{+0.4}$ \\
\hline 9 & SS $2 \ell, H_{T}>400, E_{T}>120$ & 1 & $2.3 \pm 1.2$ \\
\hline 10 & OS $2 \ell, H_{T}>300, E_{T}>275$ & 8 & $4.2 \pm 1.3$ \\
\hline
\end{tabular}

Table 2. LHC measurements used in the current study. The $\alpha_{T}$ variable is effective in suppressing background from light-quark QCD. SS $2 \ell$, and OS $2 \ell$ denote same-sign and opposite-sign dileptons, respectively. The $\alpha_{T}$ [14], SS [15], and OS [16] results were published by the CMS Collaboration.

partitioning allows us to assess the impact of the current LHC results on the pMSSM parameter space while being consistent with other existing constraints.

The prior $\pi(\theta)$ is constructed as follows. We construct the joint likelihood function of the seven independent preLHC measurements $D \equiv D_{1}, \cdots, D_{7}$, of the associated observables $\mu \equiv \mu_{1}, \cdots, \mu_{7}$, listed in table 1 . From Bayes theorem, with a flat prior $\pi(\mu)=$ constant $^{3}$ for each of the seven observables, we obtain the posterior density $p(\mu \mid D)=L(D \mid \mu) \pi(\mu) / p(D)$ from which we create a random sample of $1.5 \times 10^{7} \mathrm{pMSSM}$ parameter points using a standard MCMC technique. During the sampling, we impose the constraints on the mass, $m_{h}$, of the light neutral Higgs boson (given in row 8 of table 1 ) and the SUSY mass limits (row 9). Moreover, as we cannot scan over an infinite volume, we restrict the sampling to the sub-space $\left|M_{i}\right|,|\mu|, m_{A}, m_{\tilde{F}} \leq 3 \mathrm{TeV},\left|A_{t, b, \tau}\right| \leq 7 \mathrm{TeV}$, and $2 \leq \tan \beta \leq 60 .{ }^{4}$ The MCMC sampling from $p(\mu \mid D)$ together with the predictions $\mu_{i}=f_{i}(\theta)$ induce a distribution over $\theta$ that we take as our prior over $\theta$. By construction, the resulting set of pMSSM points are automatically consistent with the preLHC experimental constraints listed in table 1 .

From the $1.5 \times 10^{7}$ Markov-chain points we draw a subset of $5 \times 10^{5}$ points, for each of which we generate 10K events using PYTHIA6 [41]. (We checked that both the original chains

\footnotetext{
${ }^{3}$ Note that for a Gaussian density, the reference prior is flat (see, for example, ref. [40] and references therein). We will comment on prior dependence in the results section.

${ }^{4}$ Evidently, for quantities that are not well bounded by the data within the chosen sub-space, the probabilities we calculate will be somewhat sensitive to the choice of sub-space.
} 
and their subsets had converged.) We simulate the response of the CMS detector using the publicly available general purpose detector simulation package Delphes [42]. Note, that for studies of this scope, a fast, accurate, detector simulation is essential. Regarding LHC results, we use the following three published CMS SUSY analyses:

- the $\alpha_{T}$ hadronic analysis [14], based on $1.1 \mathrm{fb}^{-1}, \geq 2$ jets and $\alpha_{T}>0.55$, where $\alpha_{T}$ is used to suppress light-flavor QCD, and 8 disjoint bins in $H_{T}$, the scalar sum of jet transverse momenta;

- the same-sign (SS) di-lepton analysis [15], based on $0.98 \mathrm{fb}^{-1}$, with 8 overlapping analysis regions of which we use one, $H_{T}>400 \mathrm{GeV}$, missing transverse energy $\mathbb{E}_{T}>120 \mathrm{GeV}$, and

- the opposite-sign (OS) di-lepton analysis [16], based on $0.98 \mathrm{fb}^{-1}$, with 2 overlapping analysis regions of which we use one, $H_{T}>300 \mathrm{GeV}$ and $\not_{T}>275 \mathrm{GeV}$.

We take the observed event counts and background estimates directly from the official results of these analyses. For each of the ten results listed in table 2, we assume a Poisson likelihood,

$$
\operatorname{Poisson}\left(N_{j} \mid s_{j}+b_{j}\right)
$$

with observed count $N_{j}$ and expected count $s_{j}+b_{j}$, where $s_{j}$ and $b_{j}$ are the expected signal and background counts, respectively, for the $j^{\text {th }}$ experimental result. ${ }^{5}$ Each pMSSM point yields predictions for the values $s_{j}, j=1, \cdots, 10$. We model the (evidence-based) prior for the background parameters $b_{i}$ with a gamma density,

$$
\operatorname{gamma}\left(K_{j} b_{j} \mid Q_{j}+1\right)=e^{-K_{j} b_{j}}\left(K_{j} b_{j}\right)^{Q_{j}} / \Gamma\left(Q_{j}+1\right) .
$$

Here $Q_{j} \equiv\left(B_{j} / \delta B_{j}\right)^{2}$ and $K_{j} \equiv B_{j} / \delta B_{j}^{2}$, with $B_{j} \pm \delta B_{j}$ the background estimate in which $\delta B_{j}$ is taken to be half the width of the confidence intervals listed in table 2. For each pMSSM point, and for each result listed in table 2, we compute the (marginal) likelihood $p\left(N_{j} \mid s_{j}\right)$ by integrating over the expected background $b_{j}$. Since, by construction, the results are disjoint, the overall LHC likelihood $L(\mathrm{LHC} \mid \theta)$ is simply the product

$$
L(\mathrm{LHC} \mid \theta)=\prod_{j=1}^{10} p\left(N_{j} \mid s_{j}(\theta)\right) .
$$

The posterior density $p(\theta \mid \mathrm{LHC}) \propto L(\mathrm{LHC} \mid \theta) \pi(\theta)$ is approximated by weighting each pMSSM point by $L(\mathrm{LHC} \mid \theta)$. Finally, we normalize the posterior density over the pMSSM sub-space.

\section{Results}

We now present the results of this analysis. Figure 1 shows marginalized 1-dimensional (1D) posterior probability density functions of various sparticle and Higgs masses. The

\footnotetext{
${ }^{5}$ We use lower-case letters for parameters and upper-case letters for measured quantities.
} 
light blue histograms represent the preLHC probability densities, i.e. taking into account only the data listed in table 1 . Note that the $\tilde{\chi}_{1}^{ \pm}$and $\tilde{e}_{L} / \tilde{\mu}_{L}$ are bound to be light by the $\Delta a_{\mu}$ constraint. Note also that our preLHC distributions differ somewhat from those presented in ref. [18] as we have chosen not to impose any constraint on $\Omega h^{2}$.

The blue, green and red lines show, respectively, the effects of the OS di-lepton, SS di-lepton and $\alpha_{T}$ hadronic CMS analyses. The dashed black lines show the final posterior densities after inclusion of the results of all three analyses. It is evident that with current LHC data-sets, the di-lepton analyses have very little effect on the posterior densities, while the $\alpha_{T}$ hadronic analysis pushes the gluino and $1^{s t} / 2^{n d}$-generation squark masses towards higher values. We also note the slight effect on the $\tilde{\chi}_{1}^{0}$ LSP mass. The masses of other sparticles, including charginos, sleptons and $3^{\text {rd }}$-generation squarks, are basically unaffected by the current LHC results. This contrasts with the CMSSM case, in which all these masses are correlated through their dependence on $m_{1 / 2}$ and $m_{0}$. Finally, we see that the Higgs mass distributions, including that of $m_{h}$, remain unaffected by current SUSY searches.

The $1 \mathrm{D}$ distributions of $B R(b \rightarrow s \gamma), B R\left(B_{s} \rightarrow \mu^{+} \mu^{-}\right), \Delta a_{\mu}$ and the neutralino relic density $\Omega h^{2}$ are shown in figure 2 . In figure 3 we show the posterior densities of some SUSY Lagrangian parameters: $M_{2}, \mu, A_{t}$ and $\tan \beta$. We observe a slight preference for $\mu>0$ with $p(\mu>0) \approx 0.53$, both pre- and post-LHC startup. This is, however, inconclusive (as is the preference for $\mu<0$ found in [18]). We confirm the sign correlations between $M_{i}$ and $\mu$, and between $A_{t}$ and $\mu$, already demonstrated in ref. [18]. The corresponding plots are available at ref. [44].

It is also instructive to consider correlations between different sparticle masses. Figure 4 demonstrates the impact of the CMS analyses in the $\left(m_{\tilde{u}_{L}}, m_{\tilde{g}}\right)$ and $\left(m_{\tilde{u}_{R}}, m_{\tilde{g}}\right)$ planes. It is interesting to note that the boundaries of the LHC 95\% Bayesian credible regions (BCRs) approximately match the 95\% CL exclusion limits in the CMSSM. ${ }^{6}$ We deduce that $m_{\tilde{g}, \tilde{q}} \gtrsim 1.1 \mathrm{TeV}$ for $m_{\tilde{g}} \simeq m_{\tilde{q}}$, and $m_{\tilde{g}} \gtrsim 700 \mathrm{GeV}$ for $m_{\tilde{q}} \gg m_{\tilde{g}}$, is a robust conclusion that persists beyond the CMSSM or Simplified Models. ${ }^{7}$

Our approach moreover allows the study of dependencies between other masses in a straightforward way, as illustrated in figure 5 by means of posterior densities in the $\left(m_{\tilde{\chi}_{1}^{0}}, m_{\tilde{g}}\right)$ and $\left(m_{\tilde{\chi}_{1}^{ \pm}}, m_{\tilde{g}}\right)$ planes. We now see explicitly that bounds on the gluino mass are not reflected in chargino and neutralino masses, as would be the case in the CMSSM (or actually any scheme with gaugino-mass universality). Moreover, such plots permit other interesting observations. In particular, we see how the sensitivity of CMS searches to the gluino mass worsens for increasing neutralino or chargino mass. Additional plots of 1D and $2 \mathrm{D}$ distributions are available at ref. [44].

At this point a comment is in order regarding prior dependence. Our preLHC distributions are of course subject to the same prior dependence that was discussed in ref. [18],

\footnotetext{
${ }^{6}$ The way to calculate the BCRs is to some extent a matter of choice. Here we select the region containing the highest posterior density values. This is equivalent to choosing the minimal area that contains the $68 \%$ or $95 \%$ of the total volume.

${ }^{7}$ The characteristics of the pMSSM points with $m_{\tilde{g}}<700 \mathrm{GeV}$ that are not excluded by the current SUSY analyses will be the subject of a subsequent study; see also refs. [20, 43] in this context.
} 

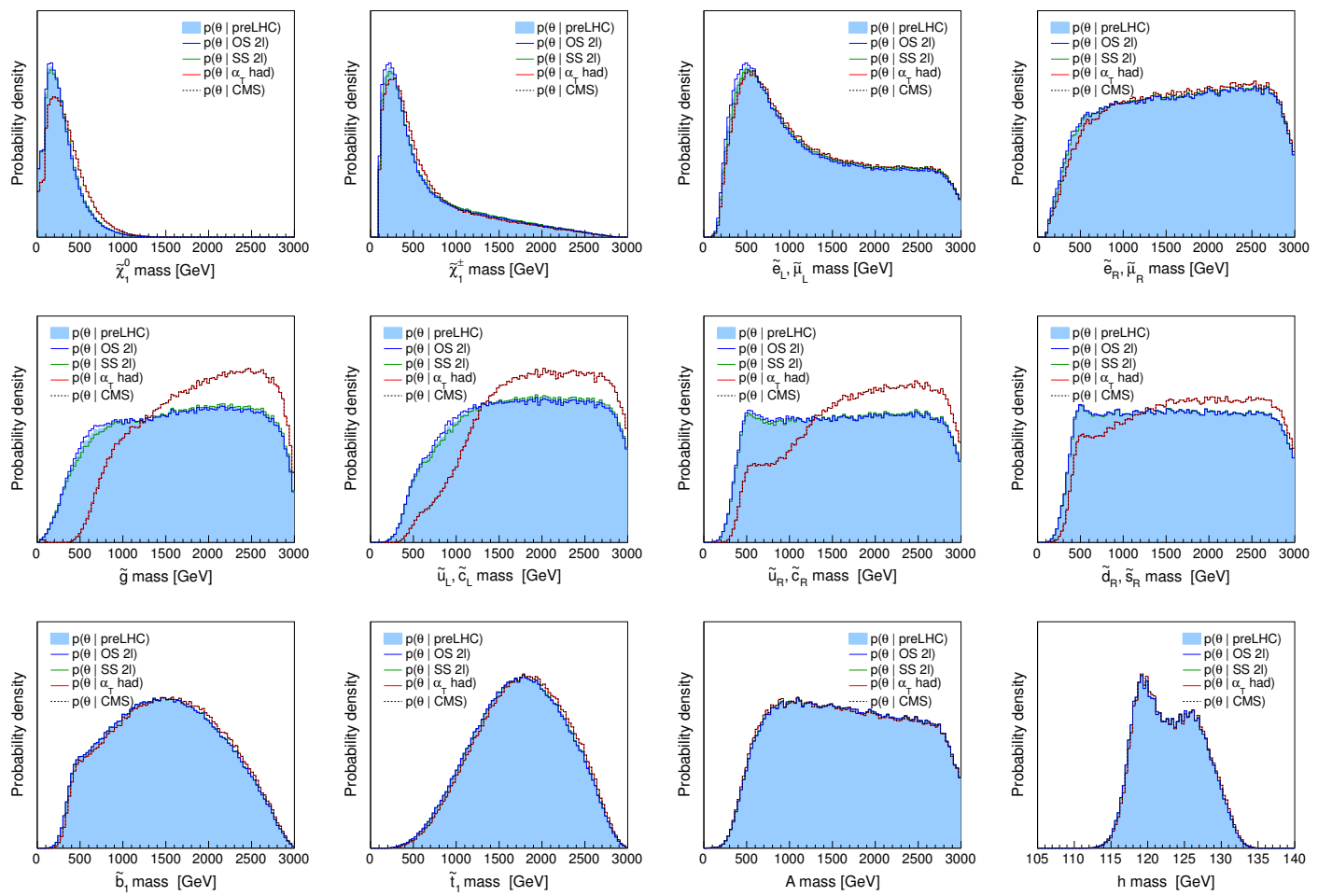

Figure 1. Marginalized 1D posterior densities of sparticle and Higgs masses.
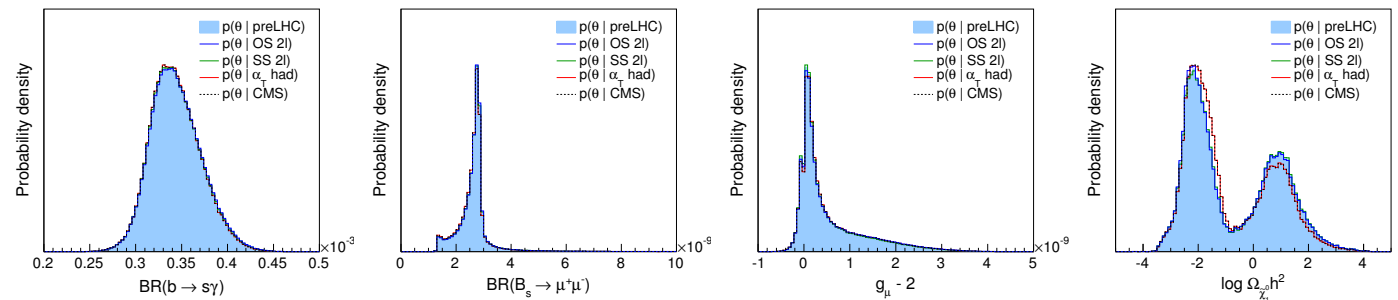

Figure 2. Marginalized 1D posterior densities of $B R(b \rightarrow s \gamma), B R\left(B_{s} \rightarrow \mu^{+} \mu^{-}\right)$, SUSY contribution to $(g-2)_{\mu}$, and neutralino relic density $\Omega h^{2}$.
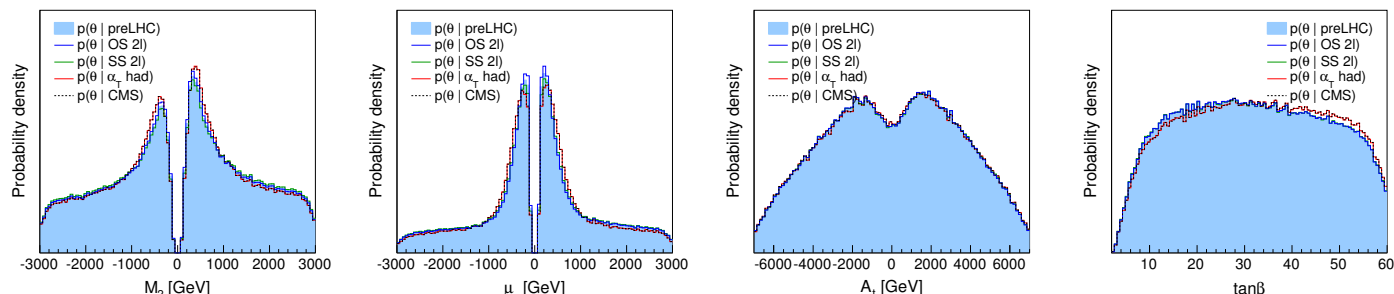

Figure 3. Marginalized 1D posterior densities for $M_{2}, \mu, A_{t}$ and $\tan \beta$.

and persists for quantities that are not much affected by the CMS measurements. However, we expect that as the effect of data becomes more influential, the sensitivity to the prior diminishes. In fact, for the gluino and $1^{\text {st }} / 2^{\text {nd }}$ generation squark masses, the likelihood based on LHC data already dominates the prior. For these quantities the posterior dis- 

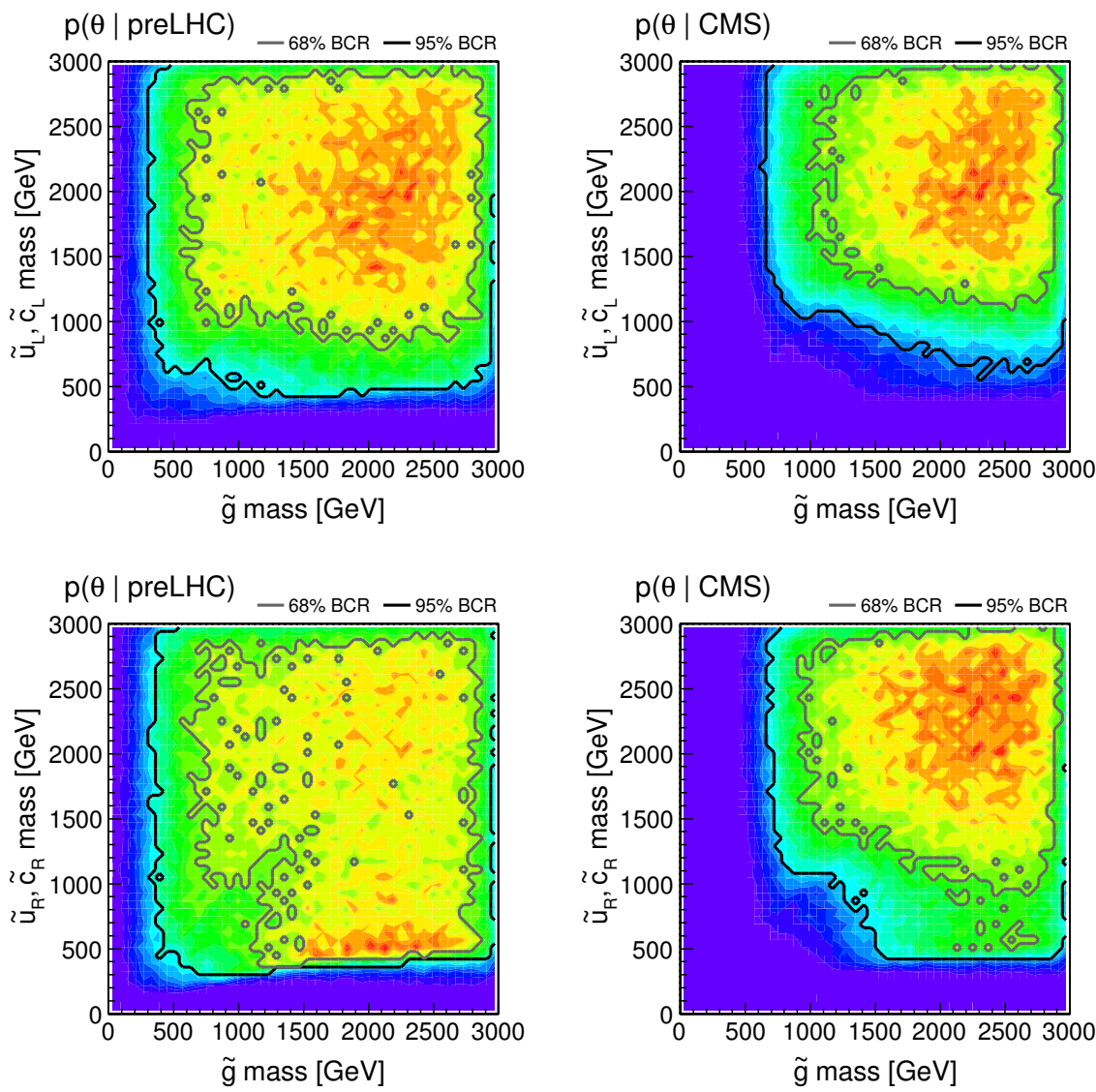

Figure 4. Marginalized 2D posterior densities of gluino versus squark masses, on the left before and on the right after taking the CMS searches into account. The grey and black contours enclose the $68 \%$ and $95 \%$ Bayesian credible regions, respectively.

tributions are indeed found to be insensitive to the choice of priors. (This is also true for some other quantities such as the $\tilde{t}_{1}$ and $h^{0}$ masses, which are already well-constrained by preLHC data.)

It is also interesting to consider the interplay with other, non-SUSY, searches. Regarding the Higgs sector, in particular the results on $H / A \rightarrow \tau \tau$ may have some impact on the pMSSM global fit. ATLAS and CMS searches for $H / A \rightarrow \tau \tau$ currently exclude $\tan \beta \lesssim 10-20$ for $m_{A} \lesssim 250 \mathrm{GeV}$, and $\tan \beta \lesssim 50-60$ for $m_{A}=450-500 \mathrm{GeV}$ [45]. While it will be interesting to include this in our global analysis, we note that our $95 \%$ BCR in the $\left(\tan \beta, m_{A}\right)$ plane, displayed in figure 6 , starts at $m_{A} \approx 500 \mathrm{GeV}$ and shows no significant dependence on $\tan \beta$.

Finally, we illustrate in figure 7 the interplay with dark matter searches. On the left, we show the posterior density in the $\left(\Omega_{\chi} h^{2}, m_{\tilde{\chi}_{1}^{0}}\right)$ plane. While matching the cosmologically observed value $\Omega h^{2}=0.1123 \pm 0.0035$ [46] needs a high degree of fine tuning, a $\tilde{\chi}_{1}^{0}$ that is at least part of the dark matter has a probability of about $60 \%$; see also the right-most plot in figure 2. In fact there is a slight increase from $p\left(\Omega_{\chi} h^{2}<0.123\right)=0.53$ with preLHC data to $p\left(\Omega_{\chi} h^{2}<0.123\right)=0.59$ when including the CMS analyses, scarcely depending on 

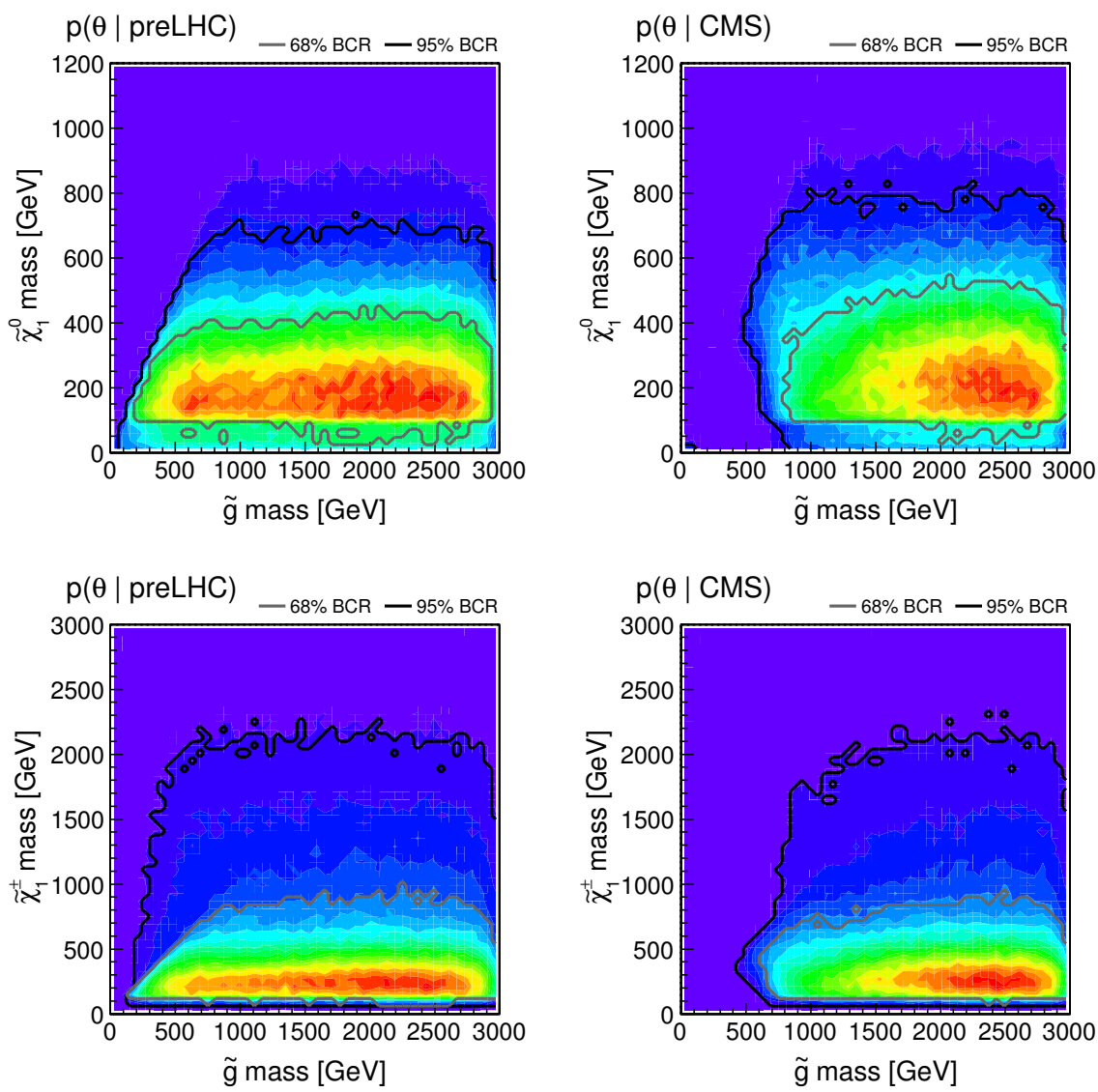

Figure 5. Marginalized 2D posterior densities of gluino versus neutralino and of gluino versus chargino mass, on the left before and on the right after taking the CMS searches into account. The grey and black contours enclose the $68 \%$ and $95 \%$ Bayesian credible regions, respectively.

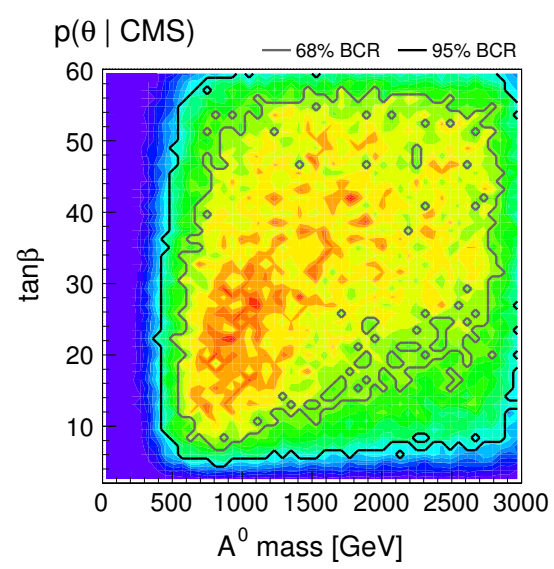

Figure 6. Marginalized 2D posterior density of $\tan \beta$ versus $m_{A}$. The grey and black contours enclose the $68 \%$ and $95 \%$ Bayesian credible regions, respectively. The preLHC distribution looks essentially the same. 

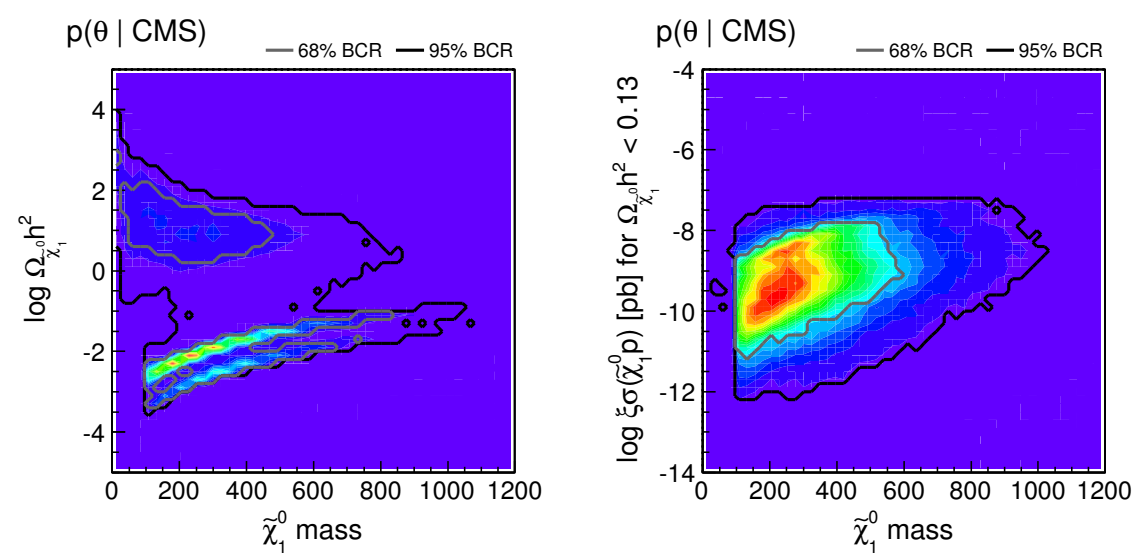

Figure 7. Marginalized 2D posterior densities of $\Omega h^{2}$ (left) and rescaled spin-independent scattering cross section off protons (right) versus LSP mass. For the latter, only points with $\Omega h^{2}<0.13$ are taken into account. The grey and black contours enclose the $68 \%$ and $95 \%$ Bayesian credible regions, respectively.

the exact value of the upper bound on $\Omega_{\chi} h^{2}$. The right plot in figure 7 shows the posterior density of the spin-independent scattering cross section off protons, $\xi \sigma^{\mathrm{SI}}\left(\tilde{\chi}_{1}^{0} p\right)$, for the case that the LSP is at least part of the dark matter. Here we imposed $\Omega h^{2}<0.13$ and rescaled the cross section by a factor $\xi=\Omega h^{2} / 0.1123$. Note that the most credible region is yet to be tested by the direct dark matter searches.

\section{Conclusions}

We presented the first interpretation of the $2011 \mathrm{LHC}$ results based on $\sim 1 \mathrm{fb}^{-1}$ of data within the framework of the phenomenological MSSM - a sufficiently generic and wellmotivated 19-dimensional parameterization of SUSY defined at the SUSY scale. We have used three independent LHC SUSY analyses, namely, the CMS $\alpha_{T}$ hadronic, opposite-sign dilepton and same-sign di-lepton analyses for this purpose, and expressed our results in terms of posterior probability densities.

Our bounds on gluino and $1^{s t} / 2^{\text {nd }}$-generation squark masses match those derived in the CMSSM by the experimental collaborations. In addition, we were able to make independent statements on the masses and properties of the other SUSY (and Higgs) particles, and to show relations between masses that weaken the current bounds. In the chargino-versusgluino-mass plane, for instance, the boundary of the $95 \%$ BCR can go down from $m_{\tilde{g}} \approx$ $800 \mathrm{GeV}$ to $m_{\tilde{g}} \approx 400 \mathrm{GeV}$, depending on the $\tilde{\chi}_{1}^{ \pm}$mass and the rest of the spectrum. Our results thus show that current SUSY searches at the LHC provide rather limited constraints on supersymmetry in general. With the currently available data and searches, we have indeed been able to probe only a small portion of the vast pMSSM parameter space, while many regions are still waiting to be explored. Being able to work constructively with generic multi-parameter models such as pMSSM will serve as a guide to identify the unexplored regions and devise a broader range of dedicated searches sensitive to these. 
We have demonstrated in this study that the interpretation of LHC results in terms of broad classes of multi-parameter SUSY models is feasible with the currently available computational and statistical tools, and that it is indeed possible to make meaningful statements on the nature of such models and therefore on supersymmetry, in general. It will be interesting to extend our study to include also results from non-SUSY searches. Indeed, one of the major advantages of our approach is that it is very well suited for global analyses of multiple results from the LHC and elsewhere.

\section{Acknowledgements}

We thank J. Hewett and T. Rizzo for discussions on "SUSY without Prejudice" and related technical issues. Moreover, we thank F. Mahmoudi and K. Williams for help with interfacing HiggsBounds, and M. Mühlleitner for fixing SUSYHIT. This work was supported in part by the U.S. Department of Energy under Grant No. DE-FG02-97ER41022 and by IN2P3 under grant PICS FR-USA 5872.

Open Access. This article is distributed under the terms of the Creative Commons Attribution License which permits any use, distribution and reproduction in any medium, provided the original author(s) and source are credited.

\section{References}

[1] G.F. Giudice, Theories for the Fermi scale, J. Phys. Conf. Ser. 110 (2008) 012014 [arXiv:0710.3294] [INSPIRE].

[2] S.P. Martin, A supersymmetry primer, hep-ph/9709356 [INSPIRE].

[3] D. Chung et al., The soft supersymmetry breaking lagrangian: theory and applications, Phys. Rept. 407 (2005) 1 [hep-ph/0312378] [INSPIRE].

[4] H. Bachacou, BSM Results from LHC, talk at Lepton-Photon 2011, August 22-27, Tata Institute of Fundamental Research, Mumbai, India (2011).

[5] A.H. Chamseddine, R.L. Arnowitt and P. Nath, Locally supersymmetric grand unification, Phys. Rev. Lett. 49 (1982) 970 [INSPIRE].

[6] R. Barbieri, S. Ferrara and C.A. Savoy, Gauge models with spontaneously broken local supersymmetry, Phys. Lett. B 119 (1982) 343 [INSPIRE].

[7] L.E. Ibáñez, Locally supersymmetric SU(5) grand unification, Phys. Lett. B 118 (1982) 73 [INSPIRE].

[8] L.J. Hall, J.D. Lykken, S. Weinberg, Supergravity as the messenger of supersymmetry breaking, Phys. Rev. D 27 (1983) 2359 [inSPIRE].

[9] P. Nath, Twenty years of SUGRA, hep-ph/0307123 [INSPIRE].

[10] G.L. Kane, C.F. Kolda, L. Roszkowski and J.D. Wells, Study of constrained minimal supersymmetry, Phys. Rev. D 49 (1994) 6173 [hep-ph/9312272] [INSPIRE].

[11] H. Baer, C.-H. Chen, R.B. Munroe, F.E. Paige and X. Tata, Multichannel search for minimal supergravity at $p \bar{p}$ and $e^{+} e^{-}$colliders, Phys. Rev. D 51 (1995) 1046 [hep-ph/9408265] [INSPIRE]. 
[12] LHC New Physics Working Group collaboration, D. Alves et al., Simplified models for LHC new physics searches, arXiv:1105.2838 [INSPIRE].

[13] MSSM Working Group collaboration, A. Djouadi et al., The minimal supersymmetric standard model: group summary report, hep-ph/9901246 [INSPIRE].

[14] CMS collaboration, S. Chatrchyan et al., Search for supersymmetry at the LHC in events with jets and missing transverse energy, Phys. Rev. Lett. 107 (2011) 221804 [arXiv: 1109.2352] [INSPIRE].

[15] CMS collaboration, Search for new physics with same-sign isolated dilepton events with jets and missing energy, PAS-SUS-11-010 (2011).

[16] CMS collaboration, Search for new physics in events with opposite-sign dileptons and missing transverse energy, PAS-SUS-11-011 (2011).

[17] C.F. Berger, J.S. Gainer, J.L. Hewett and T.G. Rizzo, Supersymmetry without prejudice, JHEP 02 (2009) 023 [arXiv:0812.0980] [INSPIRE].

[18] S.S. AbdusSalam, B.C. Allanach, F. Quevedo, F. Feroz and M. Hobson, Fitting the phenomenological MSSM, Phys. Rev. D 81 (2010) 095012 [arXiv:0904.2548] [INSPIRE].

[19] J.A. Conley, J.S. Gainer, J.L. Hewett, M.P. Le and T.G. Rizzo, Supersymmetry without prejudice at the LHC, Eur. Phys. J. C 71 (2011) 1697 [arXiv: 1009.2539] [InSPIRE].

[20] J.A. Conley, J.S. Gainer, J.L. Hewett, M.P. Le and T.G. Rizzo, Supersymmetry without prejudice at the $7 \mathrm{TeV} L H C$, arXiv:1103.1697 [INSPIRE].

[21] C.P. Robert, The Bayesian choice: from decision-theoretic foundations to computational implementation, $2^{\text {nd }}$ edition, Springer, New York U.S.A. (2007).

[22] A. O'Hagan, Bayesian inference, Kendall's Advanced Theory of Statistics volume 2B, Edward Arnold, London U.K. (1994).

[23] M. Farina et al., Implications of XENON100 and LHC results for dark matter models, Nucl. Phys. B 853 (2011) 607 [arXiv:1104.3572] [INSPIRE].

[24] B. Allanach, SOFTSUSY: a program for calculating supersymmetric spectra, Comput. Phys. Commun. 143 (2002) 305 [hep-ph/0104145] [INSPIRE].

[25] F. Mahmoudi, SuperIso v2.3: a program for calculating flavor physics observables in supersymmetry, Comput. Phys. Commun. 180 (2009) 1579 [arXiv:0808.3144] [INSPIRE].

[26] G. Bélanger, F. Boudjema, A. Pukhov and A. Semenov, MicrOMEGAs: a program for calculating the relic density in the MSSM, Comput. Phys. Commun. 149 (2002) 103 [hep-ph/0112278] [INSPIRE].

[27] G. Bélanger, F. Boudjema, A. Pukhov and A. Semenov, MicrOMEGAs: version 1.3, Comput. Phys. Commun. 174 (2006) 577 [hep-ph/0405253] [INSPIRE].

[28] G. Bélanger, F. Boudjema, A. Pukhov and A. Semenov, Dark matter direct detection rate in a generic model with MicrOMEGAs 2.2, Comput. Phys. Commun. 180 (2009) 747 [arXiv:0803.2360] [INSPIRE].

[29] P. Bechtle, O. Brein, S. Heinemeyer, G. Weiglein and K.E. Williams, HiggsBounds: confronting arbitrary Higgs sectors with exclusion bounds from LEP and the Tevatron, Comput. Phys. Commun. 181 (2010) 138 [arXiv:0811.4169] [INSPIRE]. 
[30] P. Bechtle, O. Brein, S. Heinemeyer, G. Weiglein and K.E. Williams, HiggsBounds 2.0.0: confronting neutral and charged Higgs sector predictions with exclusion bounds from LEP and the Tevatron, Comput. Phys. Commun. 182 (2011) 2605 [arXiv:1102.1898] [INSPIRE].

[31] A. Djouadi, M. Muhlleitner and M. Spira, Decays of supersymmetric particles: the program SUSY-HIT (SUspect-SdecaY-HDECAY-InTerface), Acta Phys. Polon. B 38 (2007) 635 [hep-ph/0609292] [INSPIRE].

[32] P.Z. Skands et al., SUSY Les Houches accord: Interfacing SUSY spectrum calculators, decay packages and event generators, JHEP 07 (2004) 036 [hep-ph/0311123] [INSPIRE].

[33] Heavy Flavor Averaging Group collaboration, D. Asner et al., Averages of b-hadron, c-hadron and $\tau$-lepton properties, arXiv:1010.1589 [INSPIRE].

[34] M. Misiak et al., Estimate of $B\left(\bar{B} \rightarrow X_{s} \gamma\right)$ at $O\left(\alpha_{s}^{2}\right)$, Phys. Rev. Lett. 98 (2007) 022002 [hep-ph/0609232] [INSPIRE].

[35] Particle Data Group collaboration, K. Nakamura et al., Review of particle physics, J. Phys. G 37 (2010) 075021 [INSPIRE].

[36] M. Davier, A. Hoecker, B. Malaescu and Z. Zhang, Reevaluation of the hadronic contributions to the muon $g-2$ and to $\alpha_{M Z}$, Eur. Phys. J. C 71 (2011) 1515 [arXiv: 1010.4180] [INSPIRE].

[37] CDF and D0 collaboration, T.E.W. Group, Combination of CDF and D0 results on the mass of the top quark, arXiv:0903.2503 [INSPIRE].

[38] Particle Data Group collaboration, C. Amsler et al., Review of particle physics, Phys. Lett. B 667 (2008) 1.

[39] CMS and LHCB collaboration, Search for the rare decay $B_{s}^{0} \rightarrow \mu^{+} \mu^{-}$at the LHC with the $C M S$ and LHCb experiments Combination of LHC results of the search for $B_{s} \rightarrow \mu^{+} \mu^{-}$ decays, PAS-BPH-11-019 (2011) [LHCb-CONF-2011-047] [CERN-LHCb-CONF-2011-047].

[40] L. Demortier, S. Jain and H.B. Prosper, Reference priors for high energy physics, Phys. Rev. D 82 (2010) 034002 [arXiv:1002.1111] [INSPIRE].

[41] T. Sjöstrand, S. Mrenna and P.Z. Skands, PYTHIA 6.4 physics and manual, JHEP 05 (2006) 026 [hep-ph/0603175] [INSPIRE].

[42] S. Ovyn, X. Rouby and V. Lemaitre, DELPHES, a framework for fast simulation of a generic collider experiment, arXiv:0903.2225 [INSPIRE].

[43] S. AbdusSalam, Can the LHC rule out the MSSM?, Phys. Lett. B 705 (2011) 331 [arXiv:1106.2317] [INSPIRE].

[44] http://cern.ch/kraml/plots/pMSSM.

[45] A. Nikitenko, ATLAS and CMS: BSM Higgs searches, talk at Implications of LHC results for TeV-scale physics, August 29-September 2, CERN, Switzerland (2011).

[46] N. Jarosik et al., Seven-year Wilkinson Microwave Anisotropy Probe (WMAP) observations: sky maps, systematic errors and basic results, Astrophys. J. Suppl. 192 (2011) 14 [arXiv: 1001.4744] [INSPIRE]. 